\title{
Resonance ionization schemes for high resolution and high efficiency studies of exotic nuclei at the CRIS experiment
}

Á. Koszorús ${ }^{\mathrm{a}}$, J. Billowes ${ }^{\mathrm{b}}$, C. L. Binnersley ${ }^{\mathrm{b}}$, M.L. Bissell $^{\mathrm{b}}$, T. E. Cocolios $^{\mathrm{a}}$, B.S. Cooper ${ }^{\mathrm{b}}$, R. P. de Groote ${ }^{\mathrm{a}, \mathrm{c}}$, G.J. Farooq-Smith ${ }^{\mathrm{a}}$, V.N. Fedosseev ${ }^{\mathrm{d}}$, K.

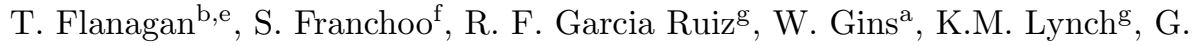
Neyens $^{\mathrm{a}, \mathrm{g}}$, F. P. Gustafsson ${ }^{\mathrm{a}}$, C. Ricketts ${ }^{\mathrm{b}}$, H.H. Stroke ${ }^{\mathrm{h}}$, A. Vernon ${ }^{\mathrm{b}}$, S.G. Wilkins $^{d}$, X. F. Yang ${ }^{\mathrm{i}}$

${ }^{a}$ KU Leuven, Instituut voor Kern- en Stralingsfysica, B-3001 Leuven, Belgium

${ }^{b}$ School of Physics and Astronomy, The University of Manchester, Manchester M13 9PL, United Kingdom

${ }^{c}$ Department of Physics, University of Jyväskylä, PB 35(YFL) FIN-40351 Jyväskylä, Finland

${ }^{d}$ Engineering Department, CERN, CH-1211 Geneva 23, Switzerland

${ }^{e}$ Photon Science Institute Alan Turing Building, University of Manchester, Manchester M13 9PY, United Kingdom

${ }^{f}$ Institut de Physique Nuclaire dOrsay, F-91406 Orsay, France

${ }^{g}$ Physics Department, CERN, CH-1211 Geneva 23, Switzerland

${ }^{h}$ Department of Physics, New York University, New York, New York 10003, USA

${ }^{i}$ School of Physics and State Key Laboratory of Nuclear Physics and Technology, Peking University, Beijing 100871, China

\begin{abstract}
This paper presents an overview of recent resonance ionization schemes used at the Collinear Resonance Ionization Spectroscopy (CRIS) setup located at ISOLDE, CERN. The developments needed to reach high spectral resolution and efficiency will be discussed. Besides laser ionization efficiency and high resolving power, experiments on rare isotopes also require low-background conditions. Ongoing developments that aim to deal with beam-related sources of background are presented.
\end{abstract}

Keywords: Resonance ionization spectroscopy, Delayed ionization,

Email address: agota.koszorus@cern.ch (Á. Koszorús) 
High-resolution laser spectroscopy, Nuclear structure,

2018 MSC: 00-01, 99-00

\section{Introduction}

The Collinear Resonance Ionization Spectroscopy (CRIS) experiment [1] was established at ISOLDE, CERN with the aim of extending the region of the nuclear chart in which high resolution laser spectroscopy can be performed. Insource RIS is currently one of the most sensitive laser spectroscopy techniques available at radioactive ion beam (RIB) facilities. This method has been used to investigate nuclear properties of isotopes produced at rates below 1 pps [2, 3]. However, since the resonance ionization is performed within the production unit, either in a hot environment or under high pressure, Doppler and/or pressure broadening of optical resonances limit the applicability of the method to isotopes which have large hyperfine splittings, such as heavier isotopes in the lead region [4, 5, 2, or specific cases such as copper or silver [3, 6, 7]. Collinear laser spectroscopy using photon detection on the other hand has superior spectral resolution due to the reduced velocity spread of the accelerated beam [8, reaching spectral resolution of the order of the natural line width. This technique has been used to study isotopes as light as ${ }^{7} \mathrm{Be}$ [9]. The sensitivity of the method has been improved using a linear Paul trap as a buncher [10, which allowed correlating the photon detection with the ion bunch arrival, and therefore reducing the photon background related to the continuous laser beam. The main limitation of the collinear laser spectroscopy using photon detection is the low efficiency compared to the RIS technique, with one photon detected in $10^{4}$ ions in most cases when measurements are performed on atoms, and 1 in a few hundred to thousands in case of ions [11, 10]. Throughout the years several modifications have successfully been implemented to improve the sensitivity of the technique [12, 13, 14, 15, 16, but they have so far only been applicable to 
specific elements.

CRIS preformed its first high resolution experiment in 2014 on francium isotopes [17]. Since then high resolution laser spectroscopy studies have been performed on 105 isotopes across 8 elements: ${ }_{88} \mathrm{Ra}\left[18\right.$, ${ }_{50} \mathrm{Sn},{ }_{49} \mathrm{In}[19],{ }_{31} \mathrm{Ga}$ [20], ${ }_{29} \mathrm{Cu}\left[21,{ }_{21} \mathrm{Sc}\right.$ and ${ }_{19} \mathrm{~K}$ isotopes, with ${ }^{232} \mathrm{Ra}$ being the heaviest and ${ }^{38} \mathrm{~K}$ the lightest isotope studied using the CRIS technique to date. In this paper the key developments and main challenges of the technique will be discussed.

\section{Experimental technique}

Isotopes studied at CRIS are produced at the ISOLDE facility at CERN by impinging protons with the energy of $1.4 \mathrm{GeV}$ onto a thick target [22]. Reaction products diffuse out of the target into a capillary tube heated to $2000{ }^{\circ} \mathrm{C}$. Surface or resonantly laser ionized isotopes are then accelerated and mass separated by the high-resolution mass separator (HRS), before being cooled and bunched in the gas-filled linear Paul trap (ISCOOL) [23]. The ion bunches with typically $2 \mu$ s temporal length are released from ISCOOL, accelerated to $30-50 \mathrm{keV}$ and guided into the CRIS beam line, schematically shown in Fig. 1. Here the ions pass through a charge-exchange cell (CEC) filled with alkali vapor at $160-320^{\circ} \mathrm{C}$. Non-neutralized ions are removed by using electrostatic deflector plates. After passing through a two stage differential pumping region (DP), the atom bunch is overlapped with laser light in a 1.2 $\mathrm{m}$ long interaction region where they are step-wise excited and ionized. This region of the beamline is held at $\sim 10^{-10}$ mbar to reduce unwanted collisional ionization. The ions are then guided towards the detection region using a $20^{\circ}$ bend, and finally detected by using a micro-channel plate (MCP Hamamatsu F4655-12) or a Magnetof (ETP MagneToF DM291) detector. Alternatively, the ions can be implanted into a simple decay spectroscopy station allowing studies of pure ground/isomeric state beams [24]. 


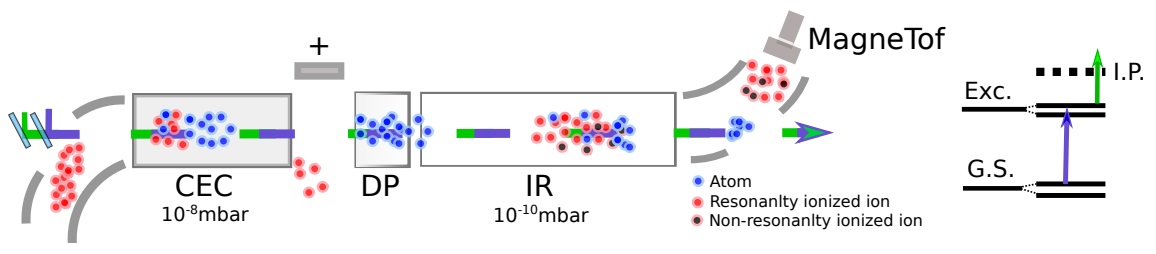

Figure 1: (Color online) (Left) Schematic representation of the CRIS beamline. The bunched beam arrives from the ISOLDE HRS mass separator and after cooling and bunching in ISCOOL is guided to the CRIS setup. See details in text. (Right) Schematic representation of an ionization scheme. The first laser light is used for resonant excitation from the ground state (G.S.) to the excited state (Exc.) and the second laser light is used for ionization from the excited state to above the ionization potential (I.P.)

The frequency of the narrow band excitation laser that is used for probing the hyperfine structure of isotopes or isotope shifts is measured using a High Finesse WSU2 wavelength meter. The absolute accuracy of this wavelength meter is specified to $2 \mathrm{MHz}$. The frequency of $1-10 \mathrm{GHz}$ bandwidth laser light is measured using a High Finesse WS6 wavelength meter with $500 \mathrm{MHz}$ absolute accuracy. As a stable frequency reference for the calibration of these devices a temperature stabilized HeNe laser has been used until 2017, after which a Toptica saturated absorption spectroscopy setup has been added. This setup enables the stabilization of a diode laser using a hyperfine transition in $\mathrm{Rb}$ or K. This diode laser was particularly important for the high-precision measurements of exotic $\mathrm{K}$ isotopes, where a close-lying calibration laser is essential in guaranteeing the performance of the wavelength meter.

To obtain a high resolution measurement of an atomic transition using the CRIS technique, the frequency of the laser light in the rest frame of the atoms is tuned by varying the frequency of the laser light, rather than varying the ion beam velocity with an accelerating or decelerating potential [25, 26], as done commonly in collinear laser spectroscopy experiments . 


\section{Resonance Ionization Schemes}

The successful study of exotic nuclei at the CRIS experiment required the realization of 4 milestones: i) achieving high resolution; ii) removing unwanted laser-atom interaction induced lineshape distortion; iii) demonstrating high selectivity and iv) high efficiency.

The first two points demand a versatile laser system and good understanding of light-atom interactions (SEC. 3.1). The CRIS experiment currently uses more than 20 lasers, including continuous wave (cw) and pulsed Titanium:Sapphire (Ti:Sa) and dye lasers and units for second (for cw light), third and fourth harmonic generation (for the pulsed lasers). The most commonly used non-resonant ionization step is provided by high pulse energy Nd:YAG lasers that operate at a repetition rate of $100 \mathrm{~Hz}$. This repetition rate determines the duty cycle of the experiment, requiring that the release of the ion bunches from ISCOOL is synchronized with the arrival of laser pulses in the interaction region.

Achieving high sensitivity imposes the requirement of an ultra high vacuum (UHV) in the interaction region and comprehensive understanding of the charge-exchange processes [27]. The UHV is required to minimize the collisional ionization within the atom bunches. In addition to this ionization mechanism, atoms can also be ionized non-resonantly by one of the high-power pulsed lasers or field ionized by the deflector plates at the $20^{\circ}$ bend. These two ionization mechanisms are particularly important for atoms in long-lived highly excited states that are produced during charge exchange.

The high total experimental efficiency is a result of optimized performance of each stage of the experiment. Firstly, the transport and bunching efficiency $\left(\varepsilon_{T R}\right)$ of the ion beam through ISCOOL (typically less than $70 \%$ ) and to the detector at the end of the CRIS beamline needs to be optimized. The typical transport efficiency of the mass separated ion beam from the entrance of the 
ISCOOL device to the detector of the CRIS beamline is less than $40 \%$, including the $70 \%$ transmission efficiency through the ISCOOL. A second source of efficiency loss is the charge-exchange process. Neutralization into the atomic state of interest occurs with an efficiency $\left(\varepsilon_{C E C}\right)$, which is strongly elementand temperature dependent. The knowledge of neutralization cross sections of the element of interest with alkali vapor at a certain beam energy, and the relative population of atomic states after the charge-exchange process is thus crucial. While the state with the highest population is preferable for RIS, a transition must exist from this state that offers sufficient sensitivity to the nuclear properties of interest. In many cases, a compromise has to be found. The operational temperature determines the density of the alkali vapor inside the CEC. A sufficiently dense vapor is required to reduce the mean free path of the ions and enable enough charge-exchange collisions, however high temperatures could result in vapor densities that allow multiple scattering of the beam and may cause energy losses resulting in lineshape distortion. Once again, the right compromise between efficiency and resolution must be sought in each atomic system.

The third component that contributes to the total efficiency is the RIS efficiency $\varepsilon_{R I S}$. This efficiency itself is the result of several aspects: laser photon density, atomic transition strengths and ionization cross-sections, spatial overlap of the laser beams with the atom beam and losses due to spontaneous decay of the atomic states [28. The atomic and laser beam have to be overlapped throughout the entire length of the interaction region. This is facilitated by using two irises with variable diameters in the differential pumping region and before the $20^{\circ}$ bend. It is crucial to have collimated laser beams with appropriate size to probe the whole ensemble of atoms in the bunch. The typical laser beam size in the interaction region is $8 \mathrm{~mm}$. By using a laser beam that is larger than the atom bunch diameter, the sensitivity to small alignment 
changes is reduced and thus increases the reliability and long-term stability of the setup. The remaining limitation to the RIS efficiency is coming from the chosen atomic transitions and photon density of the laser lights as it will be discussed in the section below. It should be noted that $\varepsilon_{C E C}$ and $\varepsilon_{R I S}$ are strongly element-dependent.

The final contribution to overall efficiency comes from the ion detection efficiency $\varepsilon_{D E T}$ of the detector (MCP or MagneTof), which is typically $80 \%$.

$$
\varepsilon=\varepsilon_{T R} \cdot \varepsilon_{C E C} \cdot \varepsilon_{R I S} \cdot \varepsilon_{D E T}
$$

Finally, when comparing efficiencies it should be noted that only atoms in one of the hyperfine levels of the ground state can be laser ionized at a time. The ratio of detected ions to the total ions thus depends on the nuclear spin of the isotope.

In summary, an overall experimental efficiency greater than $0.5 \%(1: 200)$ is possible using the CRIS technique.

\subsection{Resonance ionization spectroscopy of Fr isotopes}

Efficient RIS requires high photon flux and fluence [29, both of which can easily be produced using pulsed lasers. On the other hand, high power laser pulses can potentially introduce shifts of the atomic levels and line shape distortion [30]. The highest resolution study of exotic isotopes using the CRIS technique was performed on Fr isotopes [17, 31] using chopped cw laser light as the first excitation step. The power density of this laser was sufficient to saturate the resonant transition.

The RIS scheme is presented in Fig. 2 a). The first excitation laser light was produced by frequency doubling a cw Ti:Sa laser (Matisse TS). A power of $25 \mathrm{~mW}$ of $422.4 \mathrm{~nm}$ light was delivered to the experimental setup. Due to the long interaction time between the atoms and the cw laser light during 


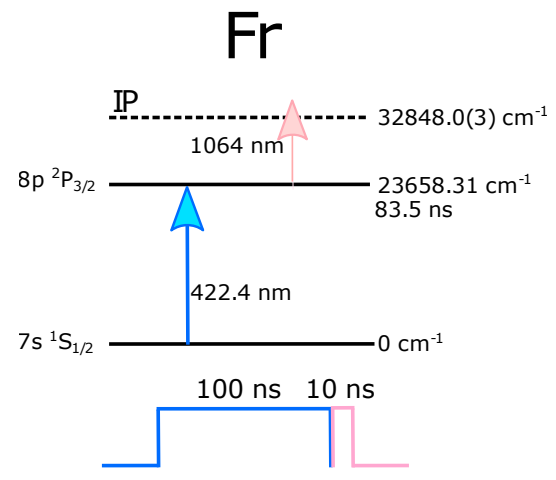

a)

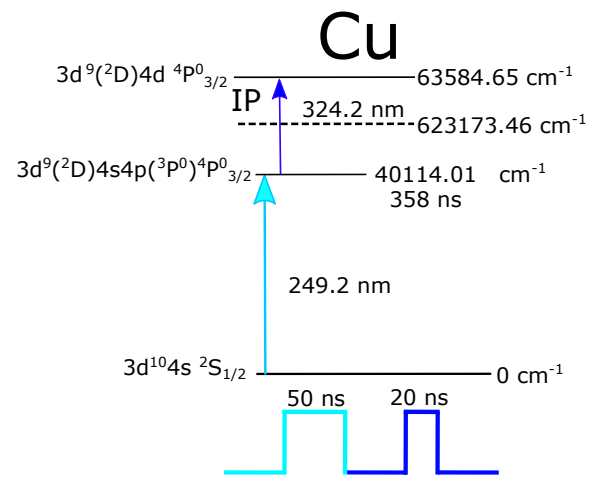

b)

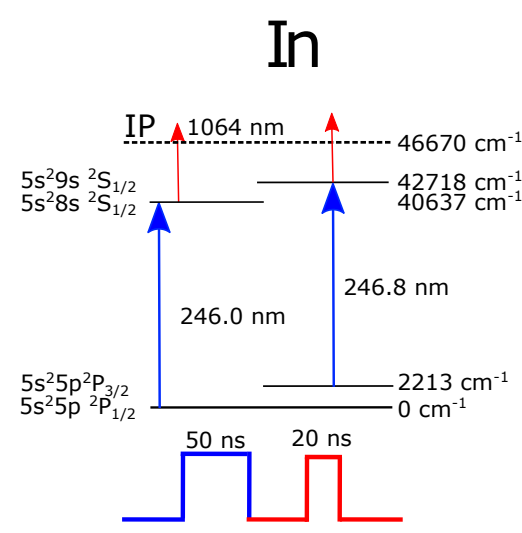

c)

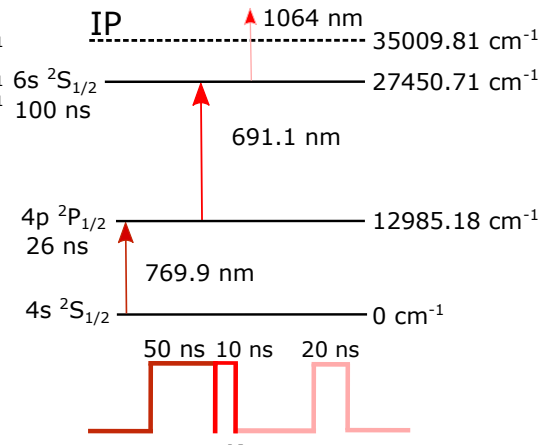

d)

Figure 2: (Color online) Resonance ionization scheme used for a) Fr, b) Cu c) In and d) $\mathrm{K}$ isotopes (top) and the sequence of laser pulses (bottom).

continuous irradiation, the population of the ground state hyperfine structure (hfs) is pumped into dark states, resulting in low RIS efficiency [32. To prevent optical pumping to such dark states, this $422.4 \mathrm{~nm}$ laser light was chopped into $100 \mathrm{~ns}$ pulses at a repetition rate of $100 \mathrm{~Hz}$ by using a Pockels cell and polarization selective optics [17. The ionization step was produced by a Litron LPY 601 50/100 PIV NdYAG laser operating at $100 \mathrm{~Hz}$.

The hfs spectrum presented in the blue histogram in Fig 3 was measured 


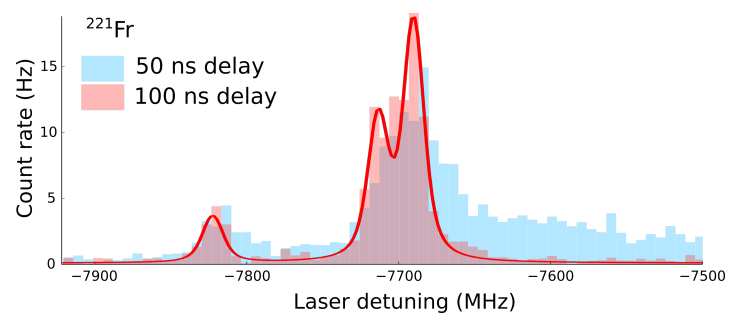

Figure 3: (Color online) Hyperfine structure of ${ }^{221} \mathrm{Fr}$ obtained with chopped cw light using different delay times for the ionization step.

by sending the chopped $\mathrm{cw}$ light into the interaction region, and the ionization laser pulse followed the excitation pulse with 50 ns delay (measured from the rising edge of the $100 \mathrm{~ns}$ excitation pulse). The line shape distortion introduced by the high power ionization step that was not temporally decoupled from the laser pulse probing the hfs is evident. Symmetric line shapes were obtained by delaying the ionization laser pulse by another $50 \mathrm{~ns}$, shown in the red histogram in Fig.3. Under these conditions, the $1064 \mathrm{~nm}$ laser pulse arrived just after the falling edge of the first laser pulse (timing sequence shown at the bottom of Fig. 22a). The width of these resonances was $20 \mathrm{MHz} F W H M$, and no significant efficiency loss was observed due to the relatively long life-time of the first excited state, which is $83.5(1.5) \mathrm{ns}$.

\subsection{Resonance ionization spectroscopy of $C u$ isotopes}

The measurement of the $\mathrm{Cu}$ isotopes in the vicinity of ${ }^{78} \mathrm{Ni}$ required high efficiency and high background suppression. It was known that the high power non-resonant ionization step introduces background. An ionization scheme that used an auto-ionizing transition was therefore used in the online experiment. This scheme offered high efficiency and sensitivity to nuclear properties 33 .

The RIS scheme for the neutron-rich $\mathrm{Cu}$ isotopes is shown in Fig. 2 b). The first state in this case required the production of UV light, most conveniently available through frequency tripling Ti:Sa light, but it can also be reached 
by frequency doubling a UV-pumped dye laser [6]. In order to ensure the saturation of this transition and achieve high resolution an injection-locked Ti:Sa laser system was installed at CRIS 34. This laser combines the narrow linewidth of a cw laser with the high power density of a pulsed laser resulting in $20 \mathrm{MHz}$ FWHM bandwidth and $360 \mu \mathrm{J}$ average pulse energy at $750 \mathrm{~nm}$. This laser was pumped by a Q-switched Nd:YAG laser (Lee Laser LDP-100MQG) at a repetition rate of $1 \mathrm{kHz}$.

The UV light that excited the first transition in $\mathrm{Cu}$ was produced by frequency tripling the fundamental output light of the injection-locked laser. The resonant ionization step to an AI state used frequency doubled light of a Spectron Pulsed Dye Laser SL4000 laser (PDL), pumped by a high-energy Nd:YAG laser (Litron TRLi 250-100) Q-Switched at a repetition rate of $100 \mathrm{~Hz}$. The advantage of using an AI state lies in the low power required for saturation. The low power density compared to the high-power non-resonant ionization with Nd:YAG lasers also reduced the probability for non-resonant laser ionization of contaminant atoms. Owing to the long life-time of the first excited state (358 ns), the ionization step could be decoupled from the excitation step without introducing efficiency losses and resulted in symmetric hyperfine peaks with a FWHM of $60 \mathrm{MHz}$. Even though the ionization step was not saturated, the overall efficiency of this experiment was calculated to be 1:100.

The 1:10 ${ }^{7}$ background suppression resulted in background free spectra and enabled the measurement of the hfs of ${ }^{78} \mathrm{Cu}$ in 8 hours with 20 pps production yield and amidst $10^{5}$ pps of isobaric contamination.

\subsection{Resonance ionization spectroscopy of In isotopes}

Indium isotopes from mass number 101 up to 131 were studied at CRIS with high resolution. With one exception, each of the In isotopes studied has at least one isomeric state. The disentanglement of the hfs peaks of different nuclear states thus provides a challenge. 
Multiple RIS schemes for the study of In isotopes were therefore carefully chosen and optimized before the experiment using an ablation ion source [19]. Firstly, the population of the ground- and metastable atomic states after the CEC was investigated and compared to calculation [35]. The ground state and the metastable state at $2213 \mathrm{~cm}^{-1}$ above it had $25 \%$ and $30 \%$ of the population, respectively, when a $20 \mathrm{kV}$ beam was neutralized using $\mathrm{Na}$ vapor. Due to a small difference in the wavelength between transitions from these states to, respectively, $9 s^{2} S_{1 / 2}$ and $8 s^{2} S_{1 / 2}$ excited states (see Fig. 2 c)), it was possible to switch from one RIS scheme to another without major intervention during the experiment. Most importantly, these two transitions have different sensitivity to nuclear properties and provide complementary information.

The RIS schemes used for measurements of neutron-deficient and -rich In isotopes are presented in Fig. 2 c). The laser light for the first resonance excitation step in both schemes was produced by frequency tripling the light of the injection-locked Ti:Sa laser. The ionization step was a $1064 \mathrm{~nm}$ Nd:YAG laser light provided by Litron TRLi 250-100 Q-Switched at a repetition rate of $100 \mathrm{~Hz}$. The high power ionization step was decoupled from the resonant laser pulse that measured the hfs just as in the case of $\mathrm{Fr}$ and $\mathrm{Cu}$, without introducing efficiency losses, since both first excited states have relatively long life-times, more than $100 \mathrm{~ns}$, compared to the $50 \mathrm{~ns}$ long laser pulse.

The main limitation during this experiment was the high background rate. Even though significant effort has been invested in improving the vacuum conditions in all sections of the CRIS beamline, reaching $10^{-10} \mathrm{mbar}$ in the interaction region, the background suppression remained unchanged at 1:107.

It was found that the most significant part of the non-resonant background was related to atoms in Rydberg states that are populated in the CEC. These were field ionized (using a $5 \mathrm{kV} / \mathrm{cm}$ electric field gradient) and removed from the beam with the help of deflector plates just after the CEC. The high power 
$1064 \mathrm{~nm}$ laser also introduced ions unrelated to the resonance excitation step. To remove the contamination that contributed to the background through this process, another $1064 \mathrm{~nm}$ laser pulse was sent through the beamline before the resonance ionization steps. This ionized a fraction of the contaminants and allowed them to be deflected before reaching the interaction region.

This experiment highlights the importance of understanding the composition of the beam and the production of non-resonantly ionized species that contribute to the background. Even with relatively high overall experimental efficiency, 1:2000 in this case, poor background suppression can limit the range of isotopes that can be studied. As a result, the measurement of ${ }^{131} \mathrm{In}$ with $10^{3}$ pps yield was challenging in the presence of more than $10^{7}$ pps isobaric contamination.

\subsection{Resonance ionization spectroscopy of $K$ isotopes}

For the study of the hfs of neutron-rich K isotopes with less than 1000 pps production yield, high efficiency and high background suppression were also required. During the experiment, resonance ionization was performed on ${ }^{38-52} \mathrm{~K}$ using the RIS scheme presented in Fig. 22 d). The first excitation step was probed with a chopped cw laser light, similar to the case discussed in SEC 3.1. The second resonance excitation step was provided by the PDL laser and for the final non-resonant $1064 \mathrm{~nm}$ ionization pulse a Litron TRLi 250-100 laser was used.

Given the short life-time of the first excited state (26 ns), the delayed second excitation step introduced significant efficiency losses, reducing the count rate on the resonance peak by a factor of two. Furthermore, due to the small hyperfine parameters of the upper state (a few tens of $\mathrm{MHz}$ for some isotopes), the power of the first laser step had to be optimized for good resolution and therefore not the highest excitation efficiency. The life-time of the second excited step is $100 \mathrm{~ns}$ and allowed the delay of the final ionization step without 
further reducing the efficiency. The effect of the high power second excitation step and ionization step of the hfs was investigated systematically and will be published in elsewhere.

The overall efficiency of the experiment was measured to be 1:1500. The non-resonant background rate was reduced by a factor of 8 by deflecting atoms ionized from Rydberg states after the CEC, and the use of an early $1064 \mathrm{~nm}$ laser pulse further reduced the background more than 2 times at mass 41 . However, this factor depends on the composition of the beam, which is different in case of other masses. The study of ${ }^{52} \mathrm{~K}$ with 300 pps production yield required a more selective detection method due to the $10^{7}$ pps contamination rate in the beam and final background suppression of 1:107 .

Since the non-resonant background contained stable ions, and owing to the convenient nuclear half-life (110 ms) and decay scheme of ${ }^{52} \mathrm{~K}, \beta$-decay assisted laser spectroscopy was used to measure the hfs of this isotope. Thus the nonresonantly ionized stable contamination did not contribute to the background in the hfs spectra, improving the signal to background ration by a factor of 40 . For these measurements, the MagneTof detector that is normally used for ion detection was removed from the path of the ions and they were implanted into a flange at the end of the CRIS beamline which has a thin aluminium window. Plastic scintillators were placed behind the aluminum flange to detect high energy $\beta$ particles following the decay of implanted resonantly ionized ${ }^{52} \mathrm{~K}$.

\section{Summary and outlook}

In this paper a study on achieving high-resolution and high-efficiency RIS on radioactive ion beams was presented. At CRIS, significant efforts have been invested into improving both aspects. These developments resulted in linewidths of $20 \mathrm{MHz}$ in Fr isotopes [17, and in the successful high-resolution measurements on beams with yields as low as 20 pps 21 amongst significant 
isobaric contamination.

Furthermore the laser light atom interaction has been investigated, highlighting the importance of delayed ionization in order to avoid line shape distortion. In most cases, a long-lived first excited state could be found $\left(T_{1 / 2}>50\right.$ $\mathrm{ns}$ ), and the delayed ionization did not result in loss of efficiency. However, for the RIS of $\mathrm{K}$ isotopes, due to the short life-time of the first excited state, a reduction of a factor of 2 in the resonant ion rate was observed with the delay of the second laser step. The high power non-resonant ionization step could still be delayed, thus the line shape distortion did not appear in the spectra.

From the presented RIS studies it is clear that the use of non-resonant laser ionization is efficient, however it often introduces undesired additional background. Therefore a transition to an auto-ionizing state should be used when possible.

On the other hand, when one knows the composition of the beam delivered to the experimental setup, it is possible to prevent the unwanted ionization processes. The $1064 \mathrm{~nm}$ related background rate can be reduced by sending a laser pulse before the RIS laser steps, and therefore the ionization and the deflection of these unwanted ions takes place before the interaction region. The most significant improvement to the background suppression resulted from the removal of atoms in Rydberg states after the CEC.

It has also been shown that if ion detection does not provide sufficient signal to background ratio, there are cases in which it can be replaced by the detection of radioactive decay of the isotopes of interest [24]. This technique significantly improves the background suppression, especially in cases like ${ }^{52} \mathrm{~K}$, in which the $10^{7}$ ion/s contamination in the beam consists of stable isotopes.

In order to reduce the background rate a few future possibilities are investigated: i) resonant excitation into a Rydberg state and subsequent field ionization from these states [36]; ii) use of energy filters to differentiate be- 
tween resonantly ionized and collisional ions, exploiting the energy difference of these ions. These developments will significantly improve the selectivity and the background suppression of the technique, allowing the study of isotopes that are produced along with high contamination.

\section{Acknowledgements}

We acknowledge the support of the ISOLDE collaboration and technical teams and the Larissa group from Johannes Gutenberg-Universität Mainz. This work was supported by the National Key R\&D Program of China (Contract No: 2018YFA0404403), the National Natural Science Foundation of China (No:11875073), FNPMLS ERC Consolidator Grant no. 648381, the BriX Research Program No. P7/12, GOA 15/010 from KU Leuven, FWO-Vlaanderen (Belgium), the Science and Technology Facilities Council consolidated grant ST/F012071/1 and continuation grant ST/J000159/1, Ernest Rutherford Grant No. ST/L002868/1, the EU Horizon2020 research and innovation programme through ENSAR2 (no. 654002). We acknowledge the financial aid of the Ed Schneiderman Fund at New York University.

\section{References}

[1] K. T. Flanagan, et al., Phys. Rev. Lett. 111 (2013) 212501.

[2] B. A. Marsh, et al., Nature Physics 14 (2018) 11631167.

[3] R. Ferrer, et al., Physics Letters B 728 (2014) 191-197.

[4] H. De Witte, et al., Phys. Rev. Lett. 98 (2007) 112502.

[5] T. E. Cocolios, et al., Phys. Rev. Lett. 106 (2011) 052503.

[6] T. E. Cocolios, et al., Phys. Rev. C 81 (2010) 014314. 
[7] Yu. Kudryavtsev, et al., NIM B 297 (2013) 7 - 22.

[8] S.L. Kaufman, Opt. Commun. 17 (3) (1976) 309.

[9] A. Krieger, et al., Phys. Rev. Lett. 108 (2012) 142501.

[10] A. Nieminen, et al., Phys. Rev. Lett. 88 (2002) 094801.

[11] K. T. Flanagan, et al., Journal of Physics G 39 (12) (2012) 125101.

[12] R. Neugart, et al., NIM B 17 (4) (1986) 354 - 359.

[13] R. E. Silverans, et al., NIM B 26 (4) (1987) 591 - 597.

[14] R. F. G. Ruiz, et al., Journal of Physics G 44 (4) (2017) 044003.

[15] D. T. Yordanov, et al., Phys. Rev. Lett. 108 (2012) 042504.

[16] K. Marinova, et al., Phys. Rev. C 84 (2011) 034313.

[17] R. P. de Groote, et al., Phys. Rev. Lett. 115 (2015) 132501.

[18] K. M. Lynch, et al., Phys. Rev. C 97 (2018) 024309.

[19] R. F. Garcia Ruiz, et al., Phys. Rev. X 8 (2018) 041005.

[20] G. J. Farooq-Smith, et al., Phys. Rev. C 96 (2017) 044324.

[21] R. P. de Groote, et al., Phys. Rev. C 96 (2017) 041302(R).

[22] R. Catherall, et al., Journal of Physics G 44 (9) (2017) 094002.

[23] H. Franberg, et al., Nucl. Instrum. Meth. B 266 (2008) 4502.

[24] K. M. Lynch, et al., Phys. Rev. X 4 (2014) 011055.

[25] K. Minamisono, et al., Phys. Rev. Lett. 117 (2016) 252501.

[26] R. Neugart, et al., J. Phys. G: Nucl. Part. Phys. 44 (2017) 064002. 
[27] C. Ryder, et al., Spectrochimica Acta Part B 113 (2015) 16-21.

[28] V.N. Fedosseev, et al., Physica Scripta 85 (5) (2012) 058104.

[29] G. S. o. Hurst, Rev. Mod. Phys. 51 (1979) 767-819.

[30] J. Bakos, Physics Reports 31 (3) (1977) 209 - 235.

[31] S. G. Wilkins, et al., Phys. Rev. C 96 (2017) 034317.

[32] R. P. de Groote, et al., Phys. Rev. A 95 (2017) 032502.

[33] R. P. de Groote, et al., Phys. Rev. A 92 (2015) 022506.

[34] V. Sonnenschein, et al., Laser Physics 27 (8) (2017) 085701.

[35] A. Vernon, et al., Spectrochimica Acta Part B: Atomic Spectroscopy 153 (2019) $61-83$.

[36] C. Schulz, et al., Journal of Physics B 24 (22) (1991) 4831. 